\title{
Ulcère gastro-duodénal et corticothérapie
}

Parmi les hormones surrénaliennes, seules les 17-hydroxy-cortico-stéroïdes (gluco-corticoïdes) possèdent une action nette sur le fonc-tionnement de l'estomac, les prednisones (derives «delta») ayant - à dose équivalente - une action plus nocive que les cortisones.

Les glucocorticoïdes aggravent Vulcère experimental produit chez Tanimal par n'importe quelle technique (sauf par derivation duo-dénale); mais cette influence est très variable, et semble se manifester soit en inhibant les reactions locales de defense, soit en jouant le role de «déclencheur» (perturbations circulatoires) plutôt qu'en stimulant la secretion gastrique. Chez le rat ou le cobaye neufs, R. Lambert (Les aspects récents de $\Gamma$ ulcère experimental; Arnette, edit. Paris 1958) produit un maximum de lesion en 15 jours à 3 semaines, mais ne réussit pas à créer d'ulcère chronique.

Etant donné le nombre des indications de la cortisone et de ses derives en thérapeutíque humaine, on pouvait prévoir que l'estomac n'y resterait pas indifferent; mais on ne peut manquer d'etre frappé par l'inégalité des proportions d'accidents ulcéreux attribués à la corticothérapie, proportion allant de $0 \%$ à $17,5 \%$ selon les series, et comportant une frequence élevée de perforations (une sur quatre) ou d'hémorragies (une sur trois).

Les circonstances d'apparition de ces lesions sont classées comme suit par F. Síguíer (Acta gastroenterologica belgica, août 1958, p. 582), selon Гexistence de: a) antecedents certains d'ulcère en evolution;

b) antecedents très éloignés d'un ulcère considéré comme guéri;

c) antecedents dyspeptiques «banaux», d'allure non-ulcéreuse; d) au-

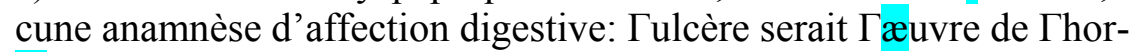

monothérapie.

Ces faits bien connus - du grand public comme des médecins - ne manquent pas d'engendrer une crainte bien comprehensible devant $\Gamma$ emploi prolongé d'une medication dont la puissance exceptionnelle est grevée de risques non négligeables; et ce n'est pas Гaddition d'un neutralisant ou d'un absorbant de l'acidité chlorhydrique ou d'un anti-sécrétoire qui préviendra tous ces accidents; la théorie de l'hyper-sécrétion chlorhydropeptique cortisonale, soutenue par Gray, n'ex-

\section{Editorial}

125

plique certainement pas tous les ulcères observes et est fortement combattue par des arguments solides.

Aussi faut-il savoir gré à L. E. Meltzer, A. A. Bookman, W. Kanen-son et A. Cohen, de Philadelphie, d'avoir étudié ce problème d'une façon systématique ("The incidence of peptic ulcer among patients on long term Prednisone therapy", Gastro-enterology d'octobre 1958, vol. 35, p. 351-356). Ils ont choisi deux groupes de malades, avant tout rhumatisants (68 sur 115), l'un traité depuis longtemps à la corticothérapie, Гautre destine à être soumis à cette medication. Le premier groupe, compose de 55 sujets ( 30 femmes et 25 hommes) de 18 à 66 ans, recevait de la Prednisone (13,8 mg. par jour en moyenne) depuis 11,2 moís. Au transit baryté, on a 
découvert 6 ulcères duodé-naux - 2 en activité, 4 chroniques - dont deux étaient connus antérieurement. Cela représente 10,9\% d'ulcéreuxparmiles malades traités; mais le tiers de ceux-ci seulement $(3,6 \%$ du total) avait vu la lesion se développer au cours de la corticothérapie. Dans le second groupe, 6 malades sur 60 étaient porteurs d'un ulcère duodenal avant Гadministration de Prednisone, dont 2 com-plétement muets, les 4 autres avec une anamnèse caractéristique. Un contrôle aux rayons $X$, après 5 mois d'hormonothérapie (14,2 mg. par jour en moyenne), ne permit plus de retrouver aucun ulcère sur les cliches!

Remarquons d'emblée que les proportions globales d'ulcères sont identiques ( $10 \%$ et $10,9 \%)$ avant le traitement (groupe 2) et après 11 mois de Prednisone (groupe 1). Mais si l'on ne retient que $\Gamma$ in-fluence de cette drogue sur la formation des ulcères, il faut conserver les chiffres de 3,6 $\%$ pour le premier groupe et de $0 \%$ pour le second, soit une moyenne de $1,7 \%$, bien inférieure aux 5 à $10 \%$ d'ulcéreux dans Tensemble de la population américaine!

Les auteurs philadelphiens admettent par consequent que leur statistique parle contre une influence de la cortisone/prednisone sur la formation d'ulcères gastro-duodénaux, d'autant plus que les arthri-tiques sont predisposes à l'ulcère $(4,5 \%)$. Allant plus loin, et pensant que l'activité cérébrale provoquée par un medicament est sans doute plus forte que celle déterminée par un «stress», ils considèrent la théorie endocrino-surrénalienne de l'étiologie de l'ulcère comme peu vraisemblable.

Cela est certes rassurant, mais ne doit pas nous enpêcher de re-doubler les precautions avec la corticothérapie appliquée aux dyspep-tiques, et bien plus encore chez les ulcéreux connus. M. Demole 